\title{
Solubilities of Benzene, Toluene and Ethylbenzene in Deep Eutectic Solvents
}

Xiaohui Qiao ${ }^{1}$, Xue Sun ${ }^{1}$, Hansen Yang ${ }^{1}$, Hualiang An 1,2, Fang Li 1,2, ${ }^{*}$, Wei Xue 1,2,*, Yanji Wang 1,2,3

1. Hebei Provincial Key Laboratory of Green Chemical Technology and High Efficient Energy Saving, School of Chemical Engineering and Technology, Hebei University of Technology, Tianjin 300130, China; 2. Tianjin Key Laboratory of Chemical Process Safety, Tianjin 300130, China; 3. Hebei Industrial Technology Research Institute of Green Chemical Industry, Huanghua 061100, Hebei, China

\footnotetext{
* Corresponding author at: School of Chemical Engineering and Technology, Hebei University of Technology, Tianjin 300130, China

E-mail: weixue@hebut.edu.cn ; lifang@hebut.edu.cn
} 

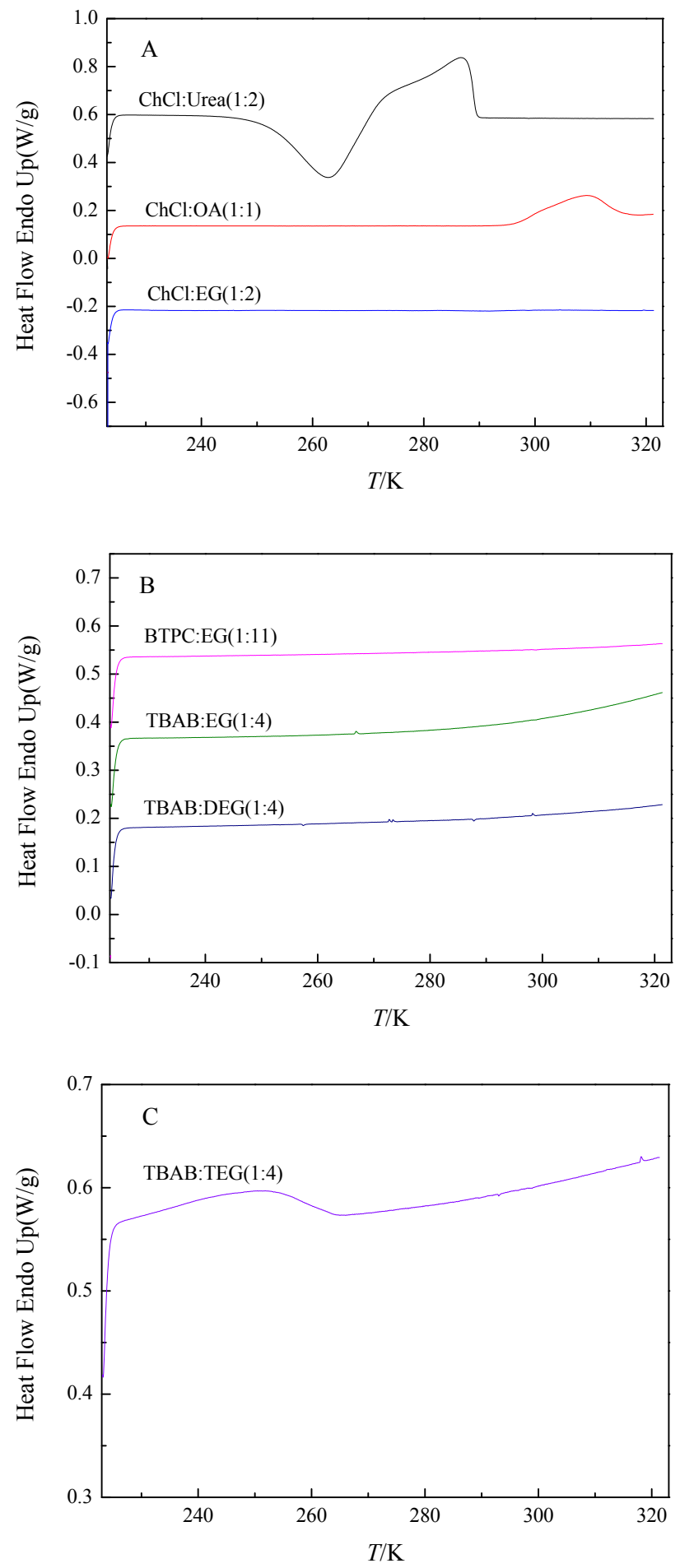

Figure S1. (A) DSC curves of ChCl: Urea (1:2), ChCl: OA (1:1) and ChCl: EG (1:2). (B) DSC curves of BTPC: EG (1:11), TBAB: EG (1:4) and TBAB: DEG (1:4). (C) DSC curve of TBAB: TEG (1:4). 
Table S1. Comparison of the Melting Point and Dynamic Viscosity $\mu$ of DESs in This Work and Data from Literatures

\begin{tabular}{cccccc}
\hline Nos. & DES & \multicolumn{2}{c}{ Melting point $\left(T_{\mathrm{m}} / \mathrm{K}\right)$} & \multicolumn{2}{c}{$\mu(\mathrm{mPa} \cdot \mathrm{s})$} \\
\hline & & & \multicolumn{2}{c}{$T$ range: $298.15-353.15 \mathrm{~K}$} \\
1 & ChCl: Urea $(1: 2)$ & $286.60^{\mathrm{a}}$ & $285.85^{\mathrm{b}}$ & $752.74-38.64^{\mathrm{a}}$ & $748.09-24.81^{\mathrm{b}}$ \\
2 & ChCl: OA $(1: 1)$ & $309.63^{\mathrm{a}}$ & $307.15^{\mathrm{c}}$ & $523.04-35.23^{\mathrm{a}}$ & --- \\
3 & ChCl: EG $(1: 2)$ & $<233.15^{\mathrm{a}}$ & $236.89^{\mathrm{b}}$ & $57.46-20.84^{\mathrm{a}}$ & $37.30-11.95^{\mathrm{b}}$ \\
4 & BTPC: EG $(1: 11)$ & $<233.15^{\mathrm{a}}$ & $248.43^{\mathrm{b}}$ & $79.35-21.35^{\mathrm{a}}$ & $51.35-11.25^{\mathrm{b}}$ \\
5 & TBAB: EG $(1: 4)$ & $<233.15^{\mathrm{a}}$ & --- & $205.66-23.63^{\mathrm{a}}$ & --- \\
6 & TBAB: DEG $(1: 4)$ & $<233.15^{\mathrm{a}}$ & --- & $346.23-28.86^{\mathrm{a}}$ & --- \\
7 & TBAB: TEG $(1: 4)$ & $251.64^{\mathrm{a}}$ & --- & $361.27-33.08^{\mathrm{a}}$ & --- \\
\hline
\end{tabular}

${ }^{a}$ Experimental data in this work. The lowest temperature detection limit of Diamond DSC is 233.15

K. Since the melting point of the sample is lower than $233.15 \mathrm{~K}$, it can not be measured. ${ }^{\mathrm{b}}$ Ref R1;

${ }^{\mathrm{c}} \operatorname{Ref} \mathrm{R} 2$.

Table S2. The Mole Fraction Solubility Data of Water in $[\mathrm{bmim}]\left[\mathrm{PF}_{6}\right]$ at Temperature $T=$ 303.15-353.15 K and Pressure $p=0.1 \mathrm{MPa}^{\text {a }}$

\begin{tabular}{cccccccc}
\hline$T / \mathrm{K}$ & $\chi^{\text {exp }}$ & $T / \mathrm{K}$ & $\chi^{b}$ & $\mathrm{RD}(\%)$ & $T / \mathrm{K}$ & $\chi^{c}$ & $\mathrm{RD}(\%)$ \\
\hline 303.15 & 0.285 & 303.15 & 0.280 & 1.75 & 303.27 & 0.313 & 9.82 \\
313.15 & 0.323 & 313.15 & 0.312 & 3.41 & 309.97 & 0.340 & 5.26 \\
323.15 & 0.362 & 323.15 & 0.363 & 0.28 & 318.62 & 0.389 & 7.46 \\
333.16 & 0.427 & 333.16 & 0.423 & 0.94 & 324.82 & 0.423 & 0.94 \\
343.15 & 0.498 & 343.15 & 0.492 & 1.20 & 340.76 & 0.508 & 2.01 \\
353.15 & 0.584 & 353.15 & 0.574 & 1.71 & 347.00 & 0.560 & 4.11 \\
\hline
\end{tabular}

${ }^{a}$ Standard uncertainties $\mathrm{u}$ are $\mathrm{u}(T)=0.02 \mathrm{~K}$ and $\mathrm{u}(p)=0.01 \mathrm{MPa}$, and relative standard uncertainty $\mathrm{u}_{\mathrm{r}}$ is $\mathrm{u}_{\mathrm{r}}(\chi)=0.02 .{ }^{\mathrm{b}} \operatorname{Ref} \mathrm{R} 3 .{ }^{\mathrm{c}} \operatorname{Ref} \mathrm{R} 4$. $\mathrm{RD}$ is the relative deviation between the experimental solubility data and the literature data, and $\mathrm{RD}=\left|\frac{\chi^{\text {exp }}-\chi}{\chi^{\exp }}\right| \times 100 \%$.

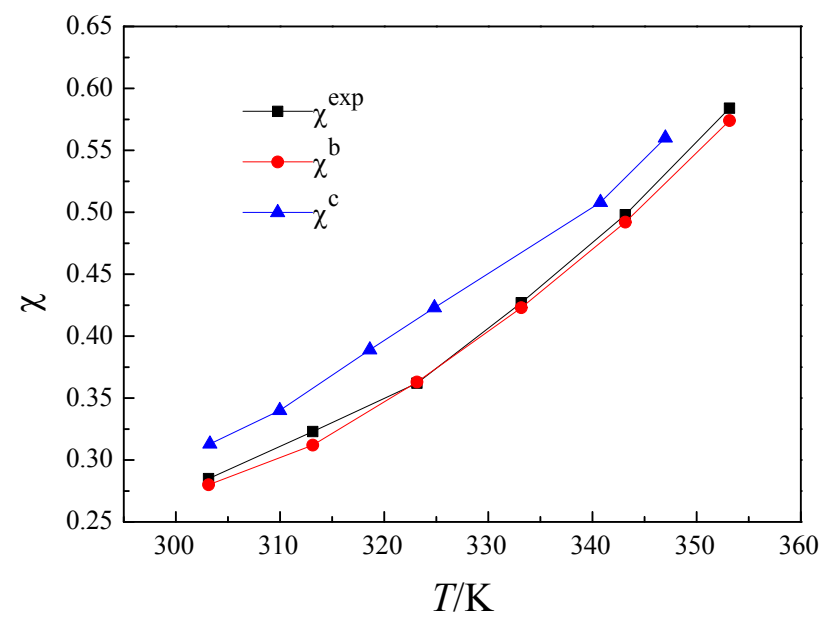

Figure S2. Comparison of the mole fraction solubility data of water in $[\mathrm{bmim}]\left[\mathrm{PF}_{6}\right]$ 
Table S3. Parameters of the Dual-parameter Equation (eq 3) and the Apelblat Equation (eq 4) Correlated from the Experimental Data of Benzene in DESs

\begin{tabular}{cccccccc}
\hline DES & \multicolumn{4}{c}{ dual-parameter equation } & \multicolumn{5}{c}{ Apelblat equation } \\
\hline & $A$ & $B$ & $\mathrm{R}^{2}$ & $A$ & $B$ & $C$ & $\mathrm{R}^{2}$ \\
ChCl: Urea (1:2) & 2.11 & -1990.50 & 0.9952 & -133.68 & 4466.88 & 20.04 & 0.9998 \\
ChCl: OA (1:1) & 3.10 & -2022.10 & 0.9710 & -304.04 & 12583.22 & 45.33 & 0.9908 \\
ChCl: EG (1:2) & 2.83 & -1762.80 & 0.8486 & -719.45 & 32584.06 & 106.59 & 0.9991 \\
BTPC: EG (1:11) & 0.10 & -548.37 & 0.9946 & -39.64 & 1341.34 & 5.86 & 0.9998 \\
TBAB: EG (1:4) & 0.76 & -516.71 & 0.9364 & -131.26 & 5761.39 & 19.48 & 0.9991 \\
TBAB: DEG (1:4) & -0.05 & -172.19 & 0.8467 & -71.00 & 3201.70 & 10.47 & 0.9983 \\
TBAB: TEG (1:4) & 0.48 & -304.40 & 0.9543 & -64.12 & 2767.89 & 9.53 & 0.9976 \\
\hline
\end{tabular}

Table S4. Parameters of the Dual-parameter Equation (eq 3) and the Apelblat Equation (eq 4) Correlated from the Experimental Data of Toluene in DESs

\begin{tabular}{cccccccc}
\hline DES & \multicolumn{4}{c}{ dual-parameter equation } & \multicolumn{5}{c}{ Apelblat equation } \\
\hline & $A$ & $B$ & $\mathrm{R}^{2}$ & $A$ & $B$ & $C$ & $\mathrm{R}^{2}$ \\
ChCl: Urea (1:2) & 3.74 & -2804.07 & 0.9876 & 222.37 & -13325.54 & -32.20 & 0.9950 \\
ChCl: OA (1:1) & 5.82 & -3223.06 & 0.9996 & 55.11 & -5595.41 & -7.26 & 0.9999 \\
ChCl: EG (1:2) & 7.25 & -3420.89 & 0.9666 & -422.14 & 17243.45 & 63.23 & 0.9849 \\
BTPC: EG (1:11) & 0.36 & -839.36 & 0.9985 & -25.41 & 400.94 & 3.80 & 0.9998 \\
TBAB: EG (1:4) & 1.18 & -771.04 & 0.9026 & -205.21 & 9161.29 & 30.39 & 0.9970 \\
TBAB: DEG (1:4) & 0.05 & -289.85 & 0.8988 & -79.83 & 3554.12 & 11.76 & 0.9990 \\
TBAB: TEG (1:4) & 0.41 & -355.86 & 0.9201 & -85.76 & 3791.25 & 12.69 & 0.9988 \\
\hline
\end{tabular}

Table S5. Parameters of the Dual-parameter Equation (eq 3) and the Apelblat Equation (eq 4) Correlated from the Experimental Data of Ethylbenzene in DESs

\begin{tabular}{cccccccc}
\hline DES & \multicolumn{4}{c}{ dual-parameter equation } & \multicolumn{5}{c}{ Apelblat equation } \\
\hline & $A$ & $B$ & $\mathrm{R}^{2}$ & $A$ & $B$ & $C$ & $\mathrm{R}^{2}$ \\
ChCl: Urea (1:2) & 4.42 & -3111.47 & 0.9976 & 62.58 & -5910.65 & -8.57 & 0.9975 \\
ChCl: OA (1:1) & 4.14 & -2811.68 & 0.9952 & 157.67 & -10200.17 & -22.61 & 0.9993 \\
ChCl: EG (1:2) & 2.92 & -2269.27 & 0.9841 & -223.73 & 8638.20 & 33.38 & 0.9977 \\
BTPC: EG (1:11) & -0.08 & -874.70 & 0.9926 & -51.33 & 1591.95 & 7.55 & 0.9967 \\
TBAB: EG (1:4) & 0.41 & -678.52 & 0.9054 & -178.90 & 7950.71 & 26.41 & 0.9977 \\
TBAB: DEG (1:4) & -0.32 & -305.01 & 0.9031 & -81.51 & 3602.42 & 11.96 & 0.9963 \\
TBAB: TEG (1:4) & 0.67 & -548.59 & 0.9531 & -98.77 & 4237.09 & 14.64 & 0.9982 \\
\hline
\end{tabular}

(R1) Ibrahim, R. K.; Hayyan, M.; AlSaadi, M. A.; Ibrahim, S.; Hayyan, A.; Hashim, M. A., Physical properties of ethylene glycol-based deep eutectic solvents. J. Mol. Liq. 2019, 276, 794-800.

(R2) Tang, B. K.; Row, K. H., Recent developments in deep eutectic solvents in chemical sciences. Mon. Chem. 2013, 144, (10), 1427-1454.

(R3) Li, Y.; Wang, L. S.; Cai, S. F., Mutual solubility of alkyl imidazolium hexafluorophosphate ionic liquids and water. J. Chem. Eng. Data 2010, 55, (11), 5289-5293.

(R4) Wang, S. L.; Jacquemin, J.; Husson, P.; Hardacre, C.; Gomes, M. F. C., Liquid-liquid 
miscibility and volumetric properties of aqueous solutions of ionic liquids as a function of temperature. J. Chem. Thermodyn. 2009, 41, (11), 1206-1214. 